\title{
The State Space of Perturbative Quantum Field Theory in Curved Spacetimes
}

\author{
Stefan Hollands* \\ Department of Physics, Enrico Fermi Institute, \\ University of Chicago, 5640 Ellis Ave., \\ Chicago, IL 60367, U.S.A. \\ Weihua Ruan ${ }^{\dagger}$ \\ Department of Mathematics, Computer Science and Statistics, \\ Purdue University Calumet, \\ Hammond, IN 46323, U.S.A.
}

October 27, 2018

\begin{abstract}
The space of continuous states of perturbative interacting quantum field theories in globally hyperbolic curved spacetimes is determined. Following Brunetti and Fredenhagen, we first define an abstract algebra of observables which contains the Wick-polynomials of the free field as well as their time-ordered products, and hence, by the well-known rules of perturbative quantum field theory, also the observables (up to finite order) of interest for the interacting quantum field theory. We then determine the space of continuous states on this algebra. Our result is that this space consists precisely of those states whose truncated $n$-point functions of the free field are smooth for all $n \neq 2$, and whose two-point function has the singularity structure of a Hadamard fundamental form. A crucial role in our analysis is played by the positivity property of states. On the technical side, our proof involves functional analytic methods, in particular the methods of microlocal analysis.
\end{abstract}

\footnotetext{
${ }^{*}$ Electronic mail: stefan@bert.uchicago.edu
}

${ }^{\dagger}$ Electronic mail: ruanw@calumet.purdue.edu 


\section{Introduction}

The perturbative construction of self-interacting quantum field theories in Minkowski spacetime was put on a completely rigorous mathematical footing in the works by Bogliubov, Parasiuk, Hepp, Zimmermann and other people [1] in the late sixties and early seventies. The issue of generalizing these constructions to curved spacetimes was first analyzed by Bunch and collaborators [2, 3]. These authors showed, within the context of Euclidean quantum field theory on Riemannian curved spaces, that if a theory is "perturbatively renormalizable" in flat space, then it remains so in curved space. However, while the perturbative definition of a quantum field theory on flat Euclidean space gives rise, via a "Wick rotation", to the definition of a corresponding theory on Minkowski space, no such connection holds for curved Lorentzian spacetimes, which, apart from a few special classes of spacetimes such as static ones, do not possess a corresponding real Riemannian section. This means that Euclidean methods cannot directly be used for the definition of interacting quantum field theories in most Lorentzian spacetimes.

Significant progress in perturbative construction of interacting quantum field theories on an arbitrary globally hyperbolic Lorentzian spacetime has recently been made by [4, 5, 6], using the mathematical tools of "microlocal analysis" [7]. In [4], the authors demonstrated that the formally infinite Wick-polynomials of a free field can be given a well-defined sense as operator-valued distributions via a normal ordering prescription. In [5], they then constructed time-ordered products of these Wick-polynomials. As in Minkowski spacetime, some "renormalization ambiguities" necessarily arise in the definition of the time-ordered products in curved spacetime, and moreover, unlike in Minkowski space, renormalization ambiguities also arise in the definition of the Wick-polynomials in curved spacetime. If one demands that these quantities be locally constructed from the metric in a generally covariant way, have a certain scaling behavior under a rescalings of the metric and have a suitable dependence under variations of the metric, then it can be shown [6], that these renormalization ambiguities are reduced to a finite number of free parameters. Moreover, a detailed analysis of the nature of these renormalization ambiguities [6] leads to the conclusion that interacting quantum field theories in globally hyperbolic spacetimes have the same classification into ones that are perturbatively renormalizable and ones that are not as in Minkowski space.

The (smeared) Wick-polynomials and their (smeared) time-ordered products may be regarded as members of some abstract ${ }^{*}$-algebra, $\mathcal{W}$. The Wick-polynomials and time-

\footnotetext{
${ }^{1}$ It turns out [6] that the normal ordered Wick-polynomials and their time-ordered products defined in 4. 50 necessarily fail to be locally constructed out of the metric in a covariant manner. A construction of Wick-polynomials that are locally defined in terms of the metric in a covariant manner and have the above additional properties was given in [6]. Work on a corresponding construction for the time-ordered products is in progress [8].

${ }^{2}$ While the construction of this algebra involves the choice of a quasi-free Hadamard of the corresponding free field theory, it turns out [6] that different choices for this state give rise to isomorphic
} 
ordered products in $\mathcal{W}$ which satisfy the above additional locality, covariance and scaling requirements can be used to define, via the usual perturbation expansions familiar from Minkowski space, the quantities of interest in the interacting theory. The infinite sums occurring in these perturbation expansions do not by themselves define elements of the algebra $\mathcal{W}$. However, if these sums are truncated at some arbitrary finte order, then the so obtained truncated expressions are elements of the algebra $\mathcal{W}$. This algebra therefore contains all observables of interest in the interacting theory up to an arbitrary finite order in perturbation theory.

In this work we investigate the space of quantum states on $\mathcal{W}$, that is, the space of states for the perturbatively defined interacting quantum field theory. Here, by a state we mean a linear functional $\psi: \mathcal{W} \rightarrow \mathbb{C}$ which is normalized so that $\psi(I)=1$, where "I" denotes the identity element in $\mathcal{W}$, and which is positive in the sense that $\psi\left(A^{*} A\right) \geq 0$ for any element $A$ in $\mathcal{W}$. The above algebraic notion of state is related to the usual Hilbert space notion of state, but it is more general: Given a representation of $\mathcal{W}$ on some Hilbert space, one can consider a vector or density matrix state in this Hilbert space as defining a corresponding algebraic state on $\mathcal{W}$. However, it is well-known that not all algebraic states - and not even all physically interesting ones - can be obtained in this manner from some specific Hilbert space representationf.

It was conjectured in [6] that the space of states on $\mathcal{W}$ consists precisely of those positive, normalized linear functionals $\psi$ whose truncated $n$-point functions of the free field are smooth for $n \neq 2$ and whose two-point function of the free field is of Hadamard form.

The main result of our paper (Thm. [11.1) is that this conjecture is correct with regard to the states $\psi$ on $\mathcal{W}$ that are continuous with respect to some natural topology on the algebra $\mathcal{W}$. In order to clarify the status of this continuity requirement, we note that, if no restriction at all was placed on the state $\psi$, then its $n$-point functions of the free field would be merely linear functionals on the space of testfunctions, but would not even have to be distributions, which is always assumed even in such general frameworks as the Wightman-axioms. On the other hand, the continuity requirement that we shall impose on the states under consideration will automatically imply that these $n$-point functions are at least distributions. In addition, since any element of $\mathcal{W}$ can be obtained as a limit of elements in the subalgebra $\mathcal{A}$ spanned by finite products of (smeared) free fields, a continuous state on $\mathcal{W}$ is uniquely determined by its restriction to the subalgebra $\mathcal{A}$, that is, by its $n$-point functions of the free field. This is in complete agreement with the philosophy behind the so-called "point-splitting" prescription for renormalizing

algebras. Therefore, as an abstract ${ }^{*}$-algebra, $\mathcal{W}$ is independent of that choice.

${ }^{3}$ Actually, we must restrict ourselves here to the vector or density matrix states contained in some common, dense invariant domain.

${ }^{4}$ For example, the standard thermal state at some finite temperature of the free field on Minkowski space gives rise to a state on $\mathcal{W}$. But this state cannot be regarded in any sense as arising from a density matrix state in the vacuum representation. 
Wick-products such as the stress energy operator-which is an element of $\mathcal{W}$, but not of $\mathcal{A}$-wherein one defines the action of a state on a Wick-product as the limit of the expectation values of suitable "point-split" quantities, which are elements of $\mathcal{A}$. Clearly, such a prescription implicitly involves a continuity assumption about the action of states on the algebra $\mathcal{W}$, which, as one can show, is a special case of the general continuity assumption considered in this paper.

An equivalent way to express our result is to say that only those states on the algebra $\mathcal{A}$ of free fields have a continuous extension to the algebra $\mathcal{W}$ of observables in perturbation theory whose truncated $n$-point functions are smooth for $n \neq 2$ and whose two-point function is of Hadamard form. It has long been known from the theory of renormalizing the stress energy operator that there exist many states for the free field whose action cannot be extended in a reasonable (that is, continuous) way from free fields to the stress energy operator. Our result puts this observation into a much more general perspective.

We note that our result does not hold in general for functionals on $\mathcal{W}$ which are continuous but which are not positive: One can construct continuous functionals on $\mathcal{W}$ whose truncated $n$-point functions are not smooth.

For simplicity and definiteness, we will here consider only the case of a Hermitian scalar field. However, the generalization of our results to other types of fields should be possible.

The organization of this paper is as follows: We first review the definition of the basic algebra of free fields, $\mathcal{A}$. After that, we recall the definition of the truncated $n$-point functions of a state on $\mathcal{A}$ and of Hadamard states, thereby giving a brief summary of some notions from microlocal analysis that shall be needed later on. We then briefly review the construction of the algebra $\mathcal{W}$ and recall how the topology on $\mathcal{W}$ is defined. After that, we present our main result, Thm. [II.1. The proof of that result makes up the rest of the paper. Some parts of this proof are moved to an appendix.

Acknowledgements: We would like to thank R. M. Wald for helpful discussions during the early stages of this research. We are indebted to him in particular for suggesting to us that the positivity property of states should play a crucial role in the proof of our main result. S. Hollands was supported by NFS grant PHY00-90138 to the University of Chicago. 


\section{Preliminaries}

\section{II.1 Definition of the minimal algebra $\mathcal{A}$ of observables for the free Klein-Gordon field}

A free classical Hermitian Klein-Gordon field on a curved spacetime is a real valued solution of the equation

$$
\left(\square-\xi R-m^{2}\right) \varphi=0,
$$

where $\square=|g|^{-1 / 2} \partial_{\mu}|g|^{1 / 2} g^{\mu \nu} \partial_{\nu}$ is the wave-operator in curved space, $R$ is the curvature scalar, and where $m, \xi$ are real parameters. It is known that this equation possesses unique advanced and retarded fundamental solutions on any (time-oriented) globally hyperbolic spacetime. These are determined by the equations

$$
\left(\square-\xi R-m^{2}\right) \Delta_{\mathrm{adv}}=\left(\square-\xi R-m^{2}\right) \Delta_{\mathrm{ret}}=\delta,
$$

and by the requirement that the support of $\Delta_{\text {adv }}$ respectively $\Delta_{\text {ret }}$ consists of pairs of points $\left(x_{1}, x_{2}\right)$ such that $x_{2}$ is in the causal past respectively future of $x_{1}$.

The theory of a quantized free Klein-Gordon field on globally hyperbolic spacetimes [9, 10] can be described in different ways. For our purposes, it is essential to use an algebraic approach. In this approach one starts with an abstract ${ }^{*}$-algebra, $\mathcal{A}$, of quantum observables for the free field theory. Several choices for $\mathcal{A}$ are possible. We here take $\mathcal{A}$ to be the *-algebra generated by the identity $I$ and the smeared field operators $\varphi(f)$, sometimes formally written as $\int \varphi(x) f(x) d \eta$, with the following relations:

- Linearity: $f \rightarrow \varphi(f) \in \mathcal{A}$ is complex linear.

- Klein-Gordon: The field operators satisfy the Klein-Gordon equation in the sense that $\varphi\left(\left(\square-\xi R-m^{2}\right) f\right)=0$.

- Hermiticity: The quantum field is Hermitian, $\varphi(f)^{*}=\varphi(\bar{f})$.

- Commutation Relations:

$$
\left[\varphi\left(f_{1}\right), \varphi\left(f_{2}\right)\right]=i \Delta\left(f_{1}, f_{2}\right) \cdot I
$$

where $\Delta=\Delta_{\mathrm{adv}}-\Delta_{\text {ret }}$.

We will subsequently consider more general observables than those contained in the algebra $\mathcal{A}$. We therefore refer to this algebra as the "minimal algebra".

\footnotetext{
${ }^{5}$ We note that the choice for $\mathcal{A}$ used in this paper is not the same as in [9, 10], where the authors work instead with the algebra generated by exponentiated smeared field operators. Such a choice has some advantages, but would not be convenient for our purposes.
} 


\section{II.2 States on $\mathcal{A}$}

Given a state $\psi$ on $\mathcal{A}$, one defines its " $n$-point functions", $\psi_{n}$, as the $n$ times multilinear functionals on the space of testfunctions given by

$$
\psi_{n}\left(f_{1}, f_{2}, \ldots, f_{n}\right)=\psi\left(\varphi\left(f_{1}\right) \varphi\left(f_{2}\right) \ldots \varphi\left(f_{n}\right)\right)
$$

Every state on $\mathcal{A}$ is uniquely determined by the collection of its $n$-point functions. One also defines the "truncated $n$-point functions", $\psi_{n}^{T}$, of a state $\psi$. For the first few $n$, these are given by

$$
\begin{aligned}
\psi_{1}^{T}(f)= & \psi_{1}(f) \\
\psi_{2}^{T}\left(f_{1}, f_{2}\right)= & \psi_{2}\left(f_{1}, f_{2}\right)-\psi_{1}\left(f_{1}\right) \psi_{1}\left(f_{2}\right) \\
\psi_{3}^{T}\left(f_{1}, f_{2}, f_{3}\right)= & \psi_{3}\left(f_{1}, f_{2}, f_{3}\right)-\psi_{1}\left(f_{1}\right) \psi_{2}\left(f_{2}, f_{3}\right)- \\
& \psi_{1}\left(f_{2}\right) \psi_{2}\left(f_{1}, f_{3}\right)-\psi_{1}\left(f_{3}\right) \psi_{2}\left(f_{1}, f_{2}\right)+2 \psi_{1}\left(f_{1}\right) \psi_{1}\left(f_{2}\right) \psi_{1}\left(f_{3}\right) .
\end{aligned}
$$

Their definition for general $n$ is as follows. Denote by $\mathcal{I}_{n}$ the set of partitions $P$ of the set $\{1, \ldots, n\}$ into pairwise disjoint, ordered subsets $r_{1}, \ldots, r_{j}$. If $r$ is a set in $P$, then we denote its elements by $r(1), \ldots, r(|r|)$, where $|r|$ is the number of elements in the set $r$. Note that by definition $r(i)<r(j)$ if $i<j$. With this notation, the truncated $n$-point functions are implicitly defined by

$$
\psi_{n}\left(f_{1}, f_{2}, \ldots, f_{n}\right)=\sum_{P \in \mathcal{I}_{n}} \prod_{r \in P} \psi_{n}^{T}\left(f_{r(1)}, f_{r(2)}, \ldots, f_{r(|r|)}\right) .
$$

Note that the sum always contains the term $\psi_{n}^{T}\left(f_{1}, \ldots, f_{n}\right)$ corresponding to the trivial partition consisting only of the set $\{1, \ldots, n\}$. Therefore, once the truncated $n$-point functions have been defined for $1, \ldots, n-1$, one can solve the above relation for $\psi_{n}^{T}$ in terms of $\psi_{n}$ and the lower order truncated $n$-point functions.

A state on $\mathcal{A}$ is called "quasi-free" if its truncated $n$-point functions are all zero except for $n=2$. A standard example for a quasi-free state is the vacuum state in Minkowskispace, and, more generally, all states constructed from some set of "positive frequency solutions" to the Klein-Gordon equation. It is a consequence of the definition (6) that the odd $n$-point functions of a quasi-free state vanish, and that the even $n$-point functions can be expressed solely in terms of the two-point function. These expressions can be summarized in the formulat

$$
\psi\left(e^{i \varphi(f)}\right)=e^{-\frac{1}{2} \psi_{2}(f, f)}
$$

6 Actually, expressions like $e^{i \varphi(f)}$ are not elements in our algebra $\mathcal{A}$, since this algebra contains by definition only finite sums of products of smeared free fields. What is meant by the formula below (and other similar formulas in the sequel) is the set of equalities obtained by expanding both sides of the equation in a formal power series and by equating the corresponding terms in these series. 
valid for any quasi-free state $\psi$.

A state $\psi$ on $\mathcal{A}$ is said to be of "Hadamard form" if its two-point function has no spacelike singularities, and if it locally can be written in the form

$$
\psi_{2}\left(x_{1}, x_{2}\right)=U\left(x_{1}, x_{2}\right) \sigma^{-1}+V\left(x_{1}, x_{2}\right) \ln \sigma+W\left(x_{1}, x_{2}\right) .
$$

Here, $\sigma$ is the signed, squared geodesic distance between the points $x_{1}$ and $x_{2}, U$ and $V$ are certain smooth functions defined in terms of the metric and the coupling parameters, and $W$ is a smooth function depending on the state in question. The $\epsilon$-prescription for the singular terms $\sigma^{-1}$ and $\ln \sigma$ is the same as for the usual vacuum two-point function in Minkowski space. Strictly speaking, the quantities $U, V$ and $W$ are well defined only for spacetimes which are analytic, so the above definition of Hadamard states needs to be modified in spacetimes which are only smooth. For a discussion of this and a precise formulation of the statement that "there are no spacelike singularities", we refer the reader to [10]. It is an immediate consequence of the definition that if $\psi$ and $\omega$ are two Hadamard states, then the difference between the corresponding two-point functions, $\psi_{2}-\omega_{2}$, is smooth.

There exists an alternative, equivalent characterization of Hadamard states in terms of the so-called "wave front set" of its associated two-point function, which plays an important role in this work. In order to state what this characterization is, we first recall the concept of the wave front set of a distribution. Let $u$ be a smooth function on $\mathbb{R}^{n}$ with compact support. Then it is known that the Fourier transform $\square$ of $u$ is rapidly decaying, that is, for any $N$, there is a constant $C_{N}$ such that

$$
|\widehat{u}(k)| \leq C_{N}(1+|k|)^{-N} \quad \text { for all } k \in \mathbb{R}^{n} .
$$

Let now $u$ be a compactly supported distribution. Then it is known that the Fourier transform $\widehat{u}$ is polynomially bounded in $k$. However, it is no longer true in general that $\widehat{u}$ is rapidly decaying in all directions. The directions in $k$-space, for which there exists no conic neighborhood (that is, a neighborhood which is invariant under multiplication by positive scalars) such that (9) holds, are called "singular directions of $u$ " and are denoted by $\Sigma(u)$. Note that $\Sigma(u)$ is by definition a conic set.

Let now $u$ be an arbitrary distribution on an open set $X \subset \mathbb{R}^{n}$, not necessarily of compact support. Then one can define the singular directions of $u$ near some point $x$ by localizing $u$ with a smooth function of compact support for which $\chi(x) \neq 0$, by defining $\Sigma_{\chi}(u)=\Sigma(\chi u)$. If one now shrinks the support of $\chi$ to the point $x$, then one obtains the singular directions of $u$ at the point $x$, defined as

$$
\Sigma_{x}(u)=\bigcap_{\chi(x) \neq 0} \Sigma_{\chi}(u)
$$

\footnotetext{
${ }^{7}$ Our convention for the Fourier transform is $\widehat{u}(k)=\frac{1}{(2 \pi)^{n / 2}} \int u(x) e^{+i k x} d^{n} x$.
} 
The wave front set, $\operatorname{WF}(u)$, of $u$ is just the union of all nonzero singular directions of $u$,

$$
\mathrm{WF}(u)=\left\{(x, k) \in X \times\left(\mathbb{R}^{n} \backslash\{0\}\right) \mid k \in \Sigma_{x}(u)\right\} .
$$

Note that it follows directly from the definition of the wave front set that a distribution $u$ is given by a smooth density if and only if $\mathrm{WF}(u)$ is empty. It can be demonstrated that the wave front set transforms covariantly under a change of coordinates, that is, if $\phi$ is a smooth one-to-one map on $X$, then $(\phi(x), k) \in \mathrm{WF}(u)$ is equivalent to $\left(x,[D \phi(x)]^{t} k\right) \in$ $\mathrm{WF}\left(\phi^{*} u\right)$, where $D \phi=\frac{\partial \phi}{\partial x}$, where ${ }^{t}$ means the transpose of a matrix and where $\phi^{*} u$ denotes the pull-back of a distribution, defined by analogy with the pull-back of a smooth density. This makes it possible to define in an invariant way the wave front set of a distribution on a smooth manifold $X$. The above transformation law then shows that $\mathrm{WF}(u)$ is intrinsically a conic subset of the cotangent bundle $T^{*} X$ minus its zero section. It is common to define, for every closed conic set $\Gamma$, the subspace $\mathcal{D}_{\Gamma}^{\prime}(X)=\left\{u \in \mathcal{D}^{\prime}(X) \mid \mathrm{WF}(u) \subset \Gamma\right\}$ of the space $\mathcal{D}^{\prime}(X)$ of all distributions on $X$.

Having introduced the wave front set of a distribution, we can now state the promised alternative characterization of Hadamard states: Namely, a state $\psi$ is Hadamard if the wave front set of its two-point function has the following form:

$$
\mathrm{WF}\left(\psi_{2}\right)=\left\{\left(x_{1}, k_{1} ; x_{2},-k_{2}\right) \in T^{*}(M \times M) \backslash\{0\} \mid\left(x_{1}, k_{1}\right) \sim\left(x_{2}, k_{2}\right), k_{1} \in\left(V_{+}\right)_{x_{1}}\right\} .
$$

The notation $\left(x_{1}, k_{1}\right) \sim\left(x_{2}, k_{2}\right)$ means that $x_{1}$ and $x_{2}$ can be joined by a null-geodesic and that the covectors $k_{1}$ and $k_{2}$ are cotangent and coparallel to that null-geodesic. $\left(V_{+}\right)_{x}$ denotes the closed forward lightcone in the cotangent space at the point $x$, defined as the set of all future directed timelike or null covectors in the cotangent space at $x$. The closed backward lightcone, $\left(V_{-}\right)_{x}$, is defined similarly. For later purposes, we also set $V_{ \pm}=\cup_{x \in M}\left(V_{ \pm}\right)_{x}$.

For the algebras considered in this paper, there holds the so-called GNS-theorem, which says that, given an algebraic state $\psi$ on the algebra, there is a ${ }^{*}$-representation $\pi_{\psi}$ of the algebra on a Hilbert space $\mathcal{H}_{\psi}$ containing vector $\left|\Omega_{\psi}\right\rangle$, which is determined, up to equivalence, by the relation $\psi(A)=\left\langle\Omega_{\psi}\left|\pi_{\psi}(A)\right| \Omega_{\psi}\right\rangle$, required to hold for all algebraic elements $A$. This representation is commonly called the "GNS-representation" of the state $\psi$. For the case of the algebras considered in this paper, the GNS-representations corresponding to different states are in general inequivalent.

\section{II.3 Definition of the extended algebra $\mathcal{W}$}

In the previous subsections, we have introduced a minimal algebra, $\mathcal{A}$, of observables for a free Klein-Gordon field, and we have introduced the notions of quasi-free states and of Hadamard states on this algebra. The algebra $\mathcal{A}$ contains the observables corresponding to the smeared $n$-point functions of the free field, $A=\varphi\left(f_{1}\right) \varphi\left(f_{2}\right) \ldots \varphi\left(f_{n}\right)$ (and finite 
linear combinations thereof). If one wants to define a nonlinear quantum field theory perturbatively off the free field theory, one must consider additional observables such as (smeared) Wick-polynomials of the free field and (smeared) time-ordered products of these fields. However, none of these observables are contained in $\mathcal{A}$.

In order to include these additional observables, we consider, besides the minimal algebra $\mathcal{A}$, an enlarged algebra of observables, $\mathcal{W}$, that contains $\mathcal{A}$ and that also contains, among others, elements corresponding to (smeared) Wick-polynomials of free fields and time-ordered products of these fields. The construction of the algebra $\mathcal{W}$ was first given by [5] and was later formalized in [12] for the case of Minkowski spacetime. The straightforward generalization of [12] to curved spacetimes can be found in [6]. The construction of $\mathcal{W}$ initially depends on the choice of some quasi-free Hadamard state $\omega$. One can show however [6] that different choices for $\omega$ give rise to isomorphic algebras $\mathcal{W}$, so in this sense $\mathcal{W}$ does not depend on the specific choice for $\omega$.

The observables in the interacting field theory (defined perturbatively off the free field theory) are given in terms of the well-known formal power series expansions in the coupling constant, whose coefficients are elements of the algebra $\mathcal{W}$. The infinite sums occurring in these expressions are, of course, not by themselves elements of $\mathcal{W}$. These series are believed not to converge, and are at best expected to approximate the "true, nonperturbative quantities" well only up to some finite order, after which they diverge. For this reason, one is only interested, even in principle, in the calculation of the interacting observables up to some finite order in perturbation theory anyway. The latter observables are elements of our algebra $\mathcal{W}$, and we therefore take the view that $\mathcal{W}$ should be regarded as the algebra of observables which are of interest in perturbative quantum field theory.

For the convenience of the reader, we now recall the basic steps in the definition of $\mathcal{W}$. Let $\omega$ be a quasi-free Hadamard state on the minimal $\mathcal{A}$, which we shall keep fixed for the rest of this work. The minimal algebra $\mathcal{A}$ contains the normal ordered smeared $n$-point functions of the free field, defined as

$$
: \varphi^{\otimes n}\left(\otimes_{i} f_{i}\right): \omega \equiv: \varphi\left(f_{1}\right) \varphi\left(f_{2}\right) \ldots \varphi\left(f_{n}\right):_{\omega}=\left.\frac{\partial^{n}}{i^{n} \partial t_{1} \partial t_{2} \ldots \partial t_{n}} G\left(\sum_{i} t_{i} f_{i}\right)\right|_{t_{1}=t_{2}=\cdots=0},
$$

where

$$
G(f)=e^{\frac{1}{2} \omega_{2}(f, f)} e^{i \varphi(f)} .
$$

${ }^{8}$ Actually, the fields $\varphi(f)$ appearing in the expression below should be understood as the representers of these algebraic elements in the GNS-representation of the state $\omega$. 
Explicitly,

$$
\begin{aligned}
: \varphi(f):_{\omega}= & \varphi(f) \\
: \varphi\left(f_{1}\right) \varphi\left(f_{2}\right):_{\omega}= & \varphi\left(f_{1}\right) \varphi\left(f_{2}\right)-\omega_{2}\left(f_{1}, f_{2}\right) \cdot I \\
: \varphi\left(f_{1}\right) \varphi\left(f_{2}\right) \varphi\left(f_{3}\right):_{\omega}= & \varphi\left(f_{1}\right) \varphi\left(f_{2}\right) \varphi\left(f_{3}\right)-\omega_{2}\left(f_{1}, f_{2}\right) \varphi\left(f_{3}\right)- \\
& \omega_{2}\left(f_{1}, f_{3}\right) \varphi\left(f_{2}\right)-\omega_{2}\left(f_{2}, f_{3}\right) \varphi\left(f_{1}\right)
\end{aligned}
$$

for the first few values of $n$. If $t$ is a smooth testfunction on $M^{n}$, we also define the elements

$$
A=: \varphi^{\otimes n}(t): \omega=\int: \varphi\left(x_{1}\right) \varphi\left(x_{2}\right) \ldots \varphi\left(x_{n}\right):_{\omega} t\left(x_{1}, x_{2}, \ldots, x_{n}\right) d \eta_{1} d \eta_{2} \ldots d \eta_{n}
$$

in the minimal algebra $\mathcal{A}$.

In order to obtain an algebra which is large enough to contain the observables of interest in perturbative quantum field theory, one would like to smear the fields : $\varphi^{\otimes n}:_{\omega}$ not only with smooth testfunctions $t$, but also in addition with certain compactly supported testdistributions. Now, smearing the operator-valued distributions : $\varphi^{\otimes n}:_{\omega}$ with a distribution involves taking the pointwise product of two distributions. As it is well-known, the pointwise product of two distributions is in general meaningless. While it is therefore impossible to smear the $: \varphi^{\otimes n}:_{\omega}$ with an arbitrary compactly supported distribution $t$, it turns out to be possible to smear it with distributions $t$ contained in a subclass $\mathcal{E}_{n}^{\prime}$ of the class of all compactly supported distributions (here the Hadamard property of $\omega$ enters). This subclass is most conveniently described in terms of the wave front set,

$$
\mathcal{E}_{n}^{\prime}=\left\{\text { symmetric, compactly supported distributions } t \text { on } M^{n}\right.
$$

$$
\text { with } \left.\operatorname{WF}(t) \subset T^{*} M^{n} \backslash\left(V_{+}^{n} \cup V_{-}^{n}\right)\right\} \text {. }
$$

Definition II.1. $\mathcal{W}$ is the ${ }^{*}$-algebra generated by the elements $A$ of the form (16) with $t \in \mathcal{E}_{n}^{\prime}$.

By construction, the extended algebra $\mathcal{W}$ contains the minimal algebra $\mathcal{A}$, but it also contains additional elements such as for example normal ordered Wick-powers at the same spacetime point, which are defined as follows: Let

$$
t\left(x_{1}, x_{2}, \ldots, x_{n}\right)=f\left(x_{1}\right) \delta\left(x_{1}, x_{2}, \ldots, x_{n}\right),
$$

where $f$ is a compactly supported testfunction. Then one can show that $t \in \mathcal{E}_{n}^{\prime}$. The algebraic element $: \varphi^{\otimes n}(t): \omega$ is just the smeared $n$-th normal ordered Wick power of the free field at the same spacetime point,

$$
: \varphi^{n}(f):_{\omega}=: \varphi^{\otimes n}(t):_{\omega},
$$


as previously defined in [4]. More generally, it can be shown [5], that $\mathcal{W}$ also contains time-ordered products of normal ordered Wick-products.

Using Wick's theorem, one can show that the product of two elements in $\mathcal{W}$ of the form (16) can again be written as a finite sum of elements of this form. This shows in particular that any element in $\mathcal{W}$ arises as a finite sum of elements of the form (16) with $t_{n} \in \mathcal{E}_{n}^{\prime}$ plus a multiple of the identity operator.

For later purposes, we would like to have a suitable notion of the continuity of states on the algebras $\mathcal{W}$ and $\mathcal{A}$. In order to define such a notion, we must first equip $\mathcal{W}$ (and therefore also $\mathcal{A}$ ) with a topology. In other words, we must explain what we mean by the statement that a sequence $\left\{A_{\kappa}\right\}$ of elements in $\mathcal{W}$ converges to an element $A$. Such a topology has been defined in [6], we here briefly indicate the main idea. One first defines a notion of convergence of a sequence $\left\{t_{\kappa}\right\}$ in the spaces of distributions $\mathcal{E}_{n}^{\prime}$ defined above in (17). Namely, such a sequence is said to converge to a distribution $t$ if

(a) the support of $t_{\kappa}$ is contained in some compact set $K$ for all $\kappa$,

(b) $t_{\kappa} \rightarrow t$ weakly in the sense of distributions,

(c) there is a closed conic set $\Gamma \subset T^{*} M^{n} \backslash\left(V_{+}^{n} \cup V_{-}^{n}\right)$ such that $\mathrm{WF}\left(t_{\kappa}\right) \subset \Gamma$ for all $\kappa$,

(d) for any properly supported pseudo differential operator $P$ with $\mu \operatorname{supp}(P) \cap \Gamma=\emptyset$, we have that $P t_{\kappa} \rightarrow P t$ in the sense of compactly supported smooth functions.

Remark. It is common to say that a sequence of distribtuions $\left\{t_{\kappa}\right\}$ satisfying (b) through $(\mathrm{d})$ "converges to $t$ in the sense of $\mathcal{D}_{\Gamma}^{\prime}$ ". For an explanation of the notion of a pseudo differential operator and the related technical terms appearing in item (d), we refer the reader to [7]. It can be shown that $t$ is again an element in $\mathcal{E}_{n}^{\prime}$, so these spaces are complete with respect to the above topology.

Having defined a notion of sequential convergence within the spaces $\mathcal{E}_{n}^{\prime}$, we now define a notion of sequential convergence in the algebra $\mathcal{W}$ as follows. Let $\left\{A_{\kappa}\right\}$ be a sequence of generators in $\mathcal{W}$, defined by distributions $t_{\kappa} \in \mathcal{E}_{n}^{\prime}$ as in (16). Then we say that the sequence $\left\{A_{\kappa}\right\}$ converges to an element $A$ of the form (16), if $t_{\kappa} \rightarrow t$ in $\mathcal{E}_{n}^{\prime}$. The so defined notion of covergence for the generators of $\mathcal{W}$ generalizes to arbitrary sequences in $\mathcal{W}$, because every element of this algebra can be written as a finite linear combination of the generators.

A state $\psi$ on $\mathcal{W}$ is said to be continuous, if $\psi\left(A_{\kappa}\right) \rightarrow \psi(A)$ whenever $A_{\kappa}$ converges to $A$. We note that, since the space of smooth testfunctions on $M^{n}$ is dense in the space $\mathcal{E}_{n}^{\prime}$, the algebra $\mathcal{A}$ is dense in $\mathcal{W}$ in the above topology. Therefore we have the important result that continuous states on $\mathcal{W}$ are completely determined by their restrictions to $\mathcal{A}$. If we consider sequences $\left\{t_{\kappa}\right\}$ satifying (a) through (d) with $\Gamma=\emptyset$, then we get that the $n$-point functions of a continuous state must be continuous in the Laurent-Schwartz topology on 
the space of smooth testfunctions. Thus, we find in particular that the $n$-point functions of a continuous state are distributions.

\section{The state space of $\mathcal{W}$}

The aim of this section is to characterize the space of continuous states on the algebra $\mathcal{W}$. We first note that, since $\mathcal{W} \supset \mathcal{A}$, every continuous state $\psi$ on $\mathcal{W}$ gives rise, by restriction, to a state on the minimal algebra $\mathcal{A}$ whose $n$-point functions are distributions. However, the opposite is not true, namely it is not true that every such state on $\mathcal{A}$ can be extended to a continuous state on $\mathcal{W}$. This may be seen for example by considering the smeared normal ordered Wick-power : $\varphi^{2}(f): \omega$, which is an element of $\mathcal{W}$, but which is not an element of $\mathcal{A}$. Now if $\psi$ is a state on $\mathcal{A}$ with distributional $n$-point functions, then its action - provided it can be defined - on this Wick-power must be given by the limit

$$
\psi\left(: \varphi^{2}(f): \omega\right)=\lim _{\kappa \rightarrow \infty} \int\left(\psi_{2}-\omega_{2}\right)\left(x_{1}, x_{2}\right) f\left(x_{1}\right) \delta_{\kappa}\left(x_{1}, x_{2}\right) d \eta_{1} d \eta_{2}
$$

where $\left\{\delta_{\kappa}\right\}$ is a suitable sequence of smooth functions tending to the delta-distribution. (Note that this prescription is just a reformulation of the usual "point-splitting" method, as explained for example in [9].) However, this limit will only exist and be independent of the particular choice of sequence $\left\{\delta_{\kappa}\right\}$ if the distribution $\left(\psi_{2}-\omega_{2}\right)\left(x_{1}, x_{2}\right)$ is at least continuous at $x_{1}=x_{2}$. There are many states on $\mathcal{A}$ which do not have this property and which therefore do not extend to $\mathcal{W}$.

The precise characterization of the space of continuous states on $\mathcal{W}$ is as follows:

Theorem III.1. (i) Let $\psi$ be a continuous state on $\mathcal{W}$. Then the two-point function of the free field must be of Hadamard form and the truncated $n$-point functions of the free field must be smooth for $n \neq 2$.

(ii) Conversely, if $\psi$ is a state on $\mathcal{A}$ whose two-point function is of Hadamard form and whose truncated $n$-point functions are smooth for all $n \neq 2$, then $\psi$ extends to a (necessarily unique) continuous state on $\mathcal{W}$.

\section{Remarks.}

1. The results of [5, 6] imply that any quasi-free Hadamard state on $\mathcal{A}$ can be extended to a continuous state on $\mathcal{W}$. Clearly, this is a special case of item (ii) of the above theorem, since quasi-free states by definition have vanishing truncated $n$-point functions for $n \neq 2$.

2. B. S. Kay has shown (unpublished manuscript) that the $N$-particle states with smooth mode functions in the GNS-representation of any quasi-free Hadamard state 
are Hadamard and have smooth truncated $n$-point functions for $n \neq 2$. By (ii) of our theorem, these states therefore extend to continuous states on $\mathcal{W}$.

3. On Robertson-Walker spacetimes, there exists the notion of "adiabatic vacuum states" on $\mathcal{A}$, introduced by Parker and defined in a mathematically rigorous way by 13. . The two-point function of such a state differs from that of a Hadamard state typically by a term which is a number of times differentiable, but which is not smooth [14]. By our theorem, adiabatic states therefore do not possess an extension to continuous states on $\mathcal{W}$. The same remark applies to the class of states recently introduced by Junker [15].

4. It is known [16] that all quasifree Hadamard states on $\mathcal{A}$ are "locally quasi-equivalent", that is, any quasifree Hadamard state locally arises as a density matrix state in the GNS-representation of any other such state.'] We here conjecture that, more generally, this is true for all states described in item (ii) above. In view of the above theorem, this would imply that any two continuous states on $\mathcal{W}$ are locally quasiequivalent.

5. In [四, the authors introduce a "microlocal spectrum condition", which generalizes to curved spacetimes the usual spectrum condition imposed on the $n$-point function of a field theory in the context of the Wightman-axioms. The content of this condition is to require that wave front set of the $n$-point functions of an admissible state should have a specific form. The microlocal spectrum condition is known to hold for the $n$-point functions of the free field in any quasi-free Hadamard state. It is an easy consequence of our result that the microlocal spectrum condition holds in fact for the $n$-point functions of any continuous state on $\mathcal{W}$.

6. We shall from now on only deal with continuous states. Therefore, for simplicity, whenever we speak of "states", we shall mean "continuous states".

Proof. We begin with the proof of (i). Let thus $\psi$ be a continuous state on $\mathcal{W}$. We need to show that the two-point function, $\psi_{2}$, is of Hadamard form, and that the truncated $n$-point functions, $\psi_{n}^{T}$, are all smooth, except for $n=2$. Let

$$
\Psi_{n}\left(f_{1}, f_{2}, \ldots, f_{n}\right) \equiv \psi\left(: \varphi\left(f_{1}\right) \varphi\left(f_{2}\right) \ldots \varphi\left(f_{n}\right): \omega\right) .
$$

Then we have

Lemma III.1. Let $\psi$ be a state on $\mathcal{A}$. Then the following statements are equivalent:

\footnotetext{
${ }^{9}$ Actually, in [16], the author does not work with the minimal algebra $\mathcal{A}$, but instead with the algebra generated by the exponentiated smeared fields. However, this difference is not relevant in as far as the issue of quasi-equivalence is concerned.
} 
(i) $\psi_{2}$ is Hadamard and $\psi_{n}^{T}$ are smooth for $n \neq 2$.

(ii) The distributions $\Psi_{n}$ are smooth for all $n$.

Proof. The proof is based on the following combinatorical formula, which we shall prove in the appendix:

$$
\left(\psi_{n}^{T}-\omega_{n}^{T}\right)\left(f_{1}, f_{2}, \ldots, f_{n}\right)=\left.\frac{\partial^{n}}{i^{n} \partial t_{1} \partial t_{2} \ldots \partial t_{n}} \ln \psi\left(G\left(\sum_{i} t_{i} f_{i}\right)\right)\right|_{t_{1}=t_{2}=\cdots=0},
$$

where $G(f)$ is defined in (14). If one carries out the differentiations in formula (22) and uses that the functional $f \rightarrow \psi(G(f))$ is the generating functional for the hierarchy of distributions $\left\{\Psi_{1}, \Psi_{2}, \ldots, \Psi_{n}, \ldots\right\}$, as well as the standard relation $\ln (1+x)=$ $\sum_{k \geq 1}(-1)^{k+1} x^{k} / k$, then one obtains the formula

$$
\left(\psi_{n}^{T}-\omega_{n}^{T}\right)\left(f_{1}, f_{2}, \ldots, f_{n}\right)=\sum_{P \in \mathcal{I}_{n}}(-1)^{|P|-1}(|P|-1) ! \prod_{r \in P} \Psi_{|r|}\left(f_{r(1)}, f_{r(2)}, \ldots, f_{r(|r|)}\right) .
$$

Thus, if $\Psi_{n}$ is smooth for all $n$, then so is $\psi_{n}^{T}-\omega_{n}^{T}$. For $n=1$ this means that $\psi_{1}$ is smooth. For $n=2$ this means that $\psi_{2}-\omega_{2}-\psi_{1} \otimes \psi_{1}$ is smooth, and hence that $\psi_{2}$ is Hadamard. For $n \geq 3$ this shows that $\psi_{n}^{T}$ is smooth, since $\omega_{n}^{T}=0$ for all $n \geq 3$. We have thus shown the implication $(i) \Longrightarrow(i i)$ of the Lemma. The implication $(i i) \Longrightarrow(i)$ can be shown similarly by solving (23) for $\Psi_{n}$ in terms of $\psi_{k}^{T}-\omega_{k}^{T}$ with $k \leq n$.

It thus remains to be shown that $\Psi_{n}$ is smooth for all $n$. We begin by showing that the wave front set of $\Psi_{n}$ is not arbitrary.

Lemma III.2. Let $\psi$ be a continuous state on $\mathcal{W}$. Then necessarily

$$
\mathrm{WF}\left(\Psi_{n}\right) \subset V_{+}^{n} \cup V_{-}^{n} \text { for all } n \text {. }
$$

Proof. Given in the Appendix.

In order to show that the wave front set of the distributions $\Psi_{n}$ is in fact empty, we proceed by an induction in $n$. For $n=0$ there is nothing to prove, since $\Psi_{0}=1$, which is clearly smooth. Let us therefore assume that $\Psi_{k}$ is smooth for all $k \leq n-1$. We need to prove that also $\Psi_{n}$ is smooth. For this, it is necessary to gain some information about the Fourier transform, $\widehat{\chi_{n} \Psi_{n}}\left(l_{1}, \ldots, l_{n}\right)$, in directions such that either all $l_{i}$ are in the future lightcone at some points $x_{i}$ or all $l_{i}$ are in the past light cone of some points $x_{i}$, where $\chi_{n}$ is a smooth bump function whose support is localized around the point $\left(x_{1}, \ldots, x_{n}\right)$ in the product manifold $M^{n}$. We prepare the ground with the following three lemmas.

Lemma III.3. Let $\psi$ be a state on $\mathcal{A}$. Then there holds

$$
\left|\psi_{n}\left(f_{1}, \ldots, f_{n}\right)\right|^{2} \leq \psi_{2 n}\left(f_{1}, \ldots, f_{n}, \bar{f}_{n}, \ldots, \bar{f}_{1}\right)
$$

for all $n$ and all testfunctions. 
Proof. By the the Cauchy-Schwartz inequality

$$
|\psi(A)|^{2} \leq \psi\left(A A^{*}\right)
$$

for all $A \in \mathcal{A}$. The statement of the lemma then follows by setting $A=\varphi\left(f_{1}\right) \cdots \varphi\left(f_{n}\right)$.

For the next lemma, we introduce the following notation. We denote by $P$ a partition of the set $\{1, \ldots, n\}$ into disjoint ordered pairs $\left\{\left(i_{1}, j_{1}\right), \ldots\left(i_{|P|}, j_{|P|}\right)\right\}$, meaning that $i<j$ for all $(i, j) \in P$. The number of pairs in the partition $P$ is denoted by $|P|$. The set of all such partitions for a given $n$ is denoted by $\mathcal{P}_{n}$. If $1 \leq k \leq n$, then we write $k \in P$ if the partition $P$ contains a pair $(i, j)$ such that either $k=i$ or $k=j$.

Lemma III.4. Let $\psi$ be a state on $\mathcal{A}$. Then there holds

$$
\psi_{n}\left(f_{1}, f_{2}, \ldots, f_{n}\right)=\sum_{P \in \mathcal{P}_{n}}(-1)^{|P|} \Psi_{n-2|P|}\left(\otimes_{k \notin P} f_{k}\right) \prod_{(i, j) \in P} \omega_{2}\left(f_{i}, f_{j}\right)
$$

for all testfunctions $f_{1}, f_{2}, \ldots, f_{n}$.

Proof. Recall that $G(f)$, defined in (14), is the generating functional for the Wick products : $\varphi\left(x_{1}\right) \varphi\left(x_{2}\right) \ldots \varphi\left(x_{n}\right): \omega$. Therefore $\psi(G(f))$ is the generating functional for the distributions $\Psi_{n}$. By repeatedly using the identity

$$
e^{i \varphi\left(f_{1}\right)} e^{i \varphi\left(f_{2}\right)}=e^{i \varphi\left(f_{1}+f_{2}\right)} e^{-\frac{i}{2} \Delta\left(f_{1}, f_{2}\right)},
$$

it is straightforward to calculate that

$$
\psi\left(e^{i t_{1} \varphi\left(f_{1}\right)} \ldots e^{i t_{n} \varphi\left(f_{n}\right)}\right)=\exp \left(\sum_{i<j} t_{i} t_{j} \omega_{2}\left(f_{i}, f_{j}\right)+\frac{1}{2} \sum_{i} t_{i}^{2} \omega_{2}\left(f_{i}, f_{i}\right)\right) \psi\left(G\left(\sum_{i} t_{i} f_{i}\right)\right) .
$$

Applying $(-i)^{n} \partial^{n} / \partial t_{1} \ldots \partial t_{n}$ to both sides of this equation and setting $t_{1}, \ldots, t_{n}$ to zero then yields the formula claimed in the lemma.

Lemma III.5. Let $\psi$ be a continuous state on $\mathcal{W}$, and let $n \geq 1$. Then $\operatorname{WF}\left(\psi_{2 n}\right)$ does not contain any elements of the form

$$
\left(x_{1}, k_{1}, \ldots, x_{n}, k_{n}, x_{n},-k_{n}, \ldots, x_{1},-k_{1}\right) \quad \text { with } k_{i} \in\left(V_{-}\right)_{x_{i}} \text { for all } i \text {. }
$$


Proof. As a preparation, let us start by introducing some notation. Let $\left(i_{1}, \ldots, i_{r}\right)$ be tuple of natural numbers with $1 \leq i_{1}<i_{2}<\ldots i_{r} \leq 2 n$. For each such a tuple, we define a map $\phi_{\left(i_{1}, i_{2}, \ldots, i_{r}\right)}: M^{2 n} \rightarrow M^{r}$ by

$$
\phi_{\left(i_{1}, i_{2}, \ldots, i_{r}\right)}\left(x_{1}, x_{2}, \ldots, x_{2 n}\right) \equiv\left(x_{i_{1}}, x_{i_{2}}, \ldots, x_{i_{r}}\right) .
$$

With this notation, Eq. (27) can be rewritten as

$$
\psi_{2 n}=\sum_{P \in \mathcal{P}_{2 n}}(-1)^{|P|} \phi_{\left(k_{1}, \ldots, k_{2 n-2|P|}\right)}^{*} \Psi_{n-2|P|} \cdot \prod_{(i, j) \in P} \phi_{(i, j)}^{*} \omega_{2},
$$

where $\left\{k_{1}, \ldots, k_{2 n-2|P|}\right\}$ is the set of numbers in $\{1, \ldots, 2 n\}$ which are not contained in the partition $P$, and where the pull-back of a distribution is defined by analogy with the pull-back of a smooth density. Note that the distributions $\phi_{(i, j)}^{*} \omega_{2}$ etc. are by definition distributions on $M^{2 n}$, so the products in formula (32) denote the pointwise product of distributions on $M^{2 n}$.

Using now formulas [7, I, Thms. 8.2.10 and 8.2.4] for the wave front of products and pull-backs of distributions, we get the estimate

$$
\begin{aligned}
& \mathrm{WF}\left(\psi_{2 n}\right) \subset \bigcup_{P \in \mathcal{P}_{2 n}} \mathrm{WF}\left(\phi_{\left(k_{1}, \ldots, k_{2 n-2|P|}\right)}^{*} \Psi_{n-2|P|} \cdot \prod_{(i, j) \in P} \phi_{(i, j)}^{*} \omega_{2}\right) \\
& \subset \bigcup_{P \in \mathcal{P}_{2 n}}\left[\left\{\phi_{\left(k_{1}, \ldots, k_{2 n-2|P|}\right)}^{*} \mathrm{WF}\left(\Psi_{n-2|P|}\right) \cup\{0\}\right\}+\sum_{(i, j) \in P}\left\{\phi_{(i, j)}^{*} \mathrm{WF}\left(\omega_{2}\right) \cup\{0\}\right\}\right] \\
& \subset \bigcup_{P \in \mathcal{P}_{2 n}}\left[\phi_{\left(k_{1}, \ldots, k_{2 n-2|P|}\right)}^{*}\left(V_{+}^{2 n-2|P|} \cup V_{-}^{2 n-2|P|}\right)+\sum_{(i, j) \in P} \phi_{(i, j)}^{*}\left(V_{+} \times V_{-}\right)\right],
\end{aligned}
$$

where we have used that $\mathrm{WF}\left(\omega_{2}\right) \subset V_{+} \times V_{-}$since $\omega$ is Hadamard, and that $\mathrm{WF}\left(\Psi_{k}\right) \subset$ $V_{+}^{k} \cup V_{-}^{k}$, by Lem. III.2. Hence, in order to prove the lemma, it is sufficient to demonstrate that if a vector $(\underline{x}, \underline{l}) \in T^{*} M^{2 n}$ of the form

$$
\left(x_{1}, l_{1}, \ldots, x_{2 n}, l_{2 n}\right) \equiv\left(x_{1}, k_{1}, \ldots, x_{n}, k_{n}, x_{n},-k_{n}, \ldots, x_{1},-k_{1}\right) \quad \text { with } k_{i} \in\left(V_{-}\right)_{x_{i}} \text { for all } i
$$

is in the set

$$
\phi_{\left(k_{1}, \ldots, k_{2 n-2|P|}\right)}^{*}\left(V_{+}^{2 n-2|P|} \cup V_{-}^{2 n-2|P|}\right)+\sum_{(i, j) \in P} \phi_{(i, j)}^{*}\left(V_{+} \times V_{-}\right)
$$

for some partition $P$, then $k_{i}=0$ for all $i$. So let $(\underline{x}, \underline{l})$ be in the set (35) for some $P$. This implies that 
(a) $l_{i} \in V_{-}, l_{j} \in V_{+}$for all $(i, j) \in P$.

(b) Either $l_{i} \in V_{+}$for all $i \notin P$ or $l_{i} \in V_{-}$for all $i \notin P$.

Now property (a), together with the specific form of $(\underline{x}, \underline{l})$ given by (34), implies that $l_{i}=l_{j}=0$ whenever $(i, j) \in P$. Combining this with property (b), we see that the $l_{i}$ must either be all in $V_{-}$or all in $V_{+}$. Using again the specific form of $(\underline{x}, \underline{l})$, we conclude that this is only possible when all $l_{i}=0$, implying that all $k_{i}=0$.

We have now gathered enough information to show that $\Psi_{n}$ is smooth for all $n$. By the induction hypothesis, we know that $\Psi_{k}$ is smooth for all $k \leq n-1$. We want to use this to obtain an estimate for the Fourier transform of $\chi_{n} \Psi_{n}$, where $\chi_{n}$ is a smooth function with compact support that will be specified momentarily.

From Lemmas 111.4 and 111.3 we get the inequality

$$
\begin{aligned}
& \psi_{2 n}\left(f_{1}, \ldots, f_{n}, \bar{f}_{n}, \ldots, \bar{f}_{1}\right) \geq \\
& \sum_{P, P^{\prime} \in \mathcal{P}_{n}}(-1)^{|P|+\left|P^{\prime}\right|} \Psi_{n-2|P|}\left(\otimes_{k \notin P} \bar{f}_{k}\right) \Psi_{n-2\left|P^{\prime}\right|}\left(\otimes_{k^{\prime} \notin P^{\prime}} f_{k^{\prime}}\right) \prod_{(i, j) \in P} \omega_{2}\left(\bar{f}_{j}, \bar{f}_{i}\right) \prod_{\left(i^{\prime}, j^{\prime}\right) \in P^{\prime}} \omega_{2}\left(f_{i^{\prime}}, f_{j^{\prime}}\right) .
\end{aligned}
$$

We now specialize the above inequality to testfunctions $f_{j}$ of the form

$$
f_{j}(x)=\frac{1}{(2 \pi)^{2}} \eta_{j}(x) e^{i l_{j} x} .
$$

Here, $\eta_{j}$ are real-valued smooth bump functions whose support is contained in some chart, $l_{j}$ are vectors in $\mathbb{R}^{4}$ and the expression $l_{j} x$ denotes the scalar product in $\mathbb{R}^{4}$ between $l_{j}$ and the coordinate components of $x$ in the above chart. With this choice for $f_{j}$, the above inequality can be rewritten as

$$
\begin{aligned}
& \left|\widehat{\chi_{n} \Psi_{n}}\left(l_{1}, \ldots, l_{n}\right)\right|^{2} \leq \widehat{\chi_{2 n} \psi_{2 n}}\left(l_{1}, \ldots, l_{n},-l_{n}, \ldots,-l_{1}\right)- \\
& \sum_{P, P^{\prime} \in \mathcal{P}_{n}, P, P^{\prime} \neq \emptyset}(-1)^{|P|+\left|P^{\prime}\right|} \chi_{n-2|P|} \widehat{\Psi_{n}-2|P|}\left(-l_{k_{1}}, \ldots,-l_{k_{n-2|P|}}\right) \chi_{n-2\left|P^{\prime}\right| \Psi_{n-2\left|P^{\prime}\right|}}\left(l_{k_{1}^{\prime}}, \ldots, l_{k_{n-2\left|P^{\prime}\right|}^{\prime}}\right) \\
& \times \prod_{(i, j) \in P} \widehat{\chi_{2} \omega_{2}}\left(-l_{j},-l_{i}\right) \prod_{\left(i^{\prime}, j^{\prime}\right) \in P^{\prime}} \widehat{\chi_{2} \omega_{2}}\left(l_{i^{\prime}}, l_{j^{\prime}}\right),
\end{aligned}
$$

where $\left\{k_{1}, \ldots, k_{2 n-2|P|}\right\}$ is the set of numbers in $\{1, \ldots, n\}$ which are not contained in the partition $P$ and where $\left\{k_{1}^{\prime}, \ldots, k_{2 n-2\left|P^{\prime}\right|}^{\prime}\right\}$ is the set of numbers in $\{1, \ldots, n\}$ which are not contained in the partition $P^{\prime}$. The smooth functions $\chi_{k}$ denote suitable tensor products of $k$ factors of the functions $\eta_{i}$. For example, in the expression $\widehat{\chi_{2} \omega_{2}}\left(l_{i^{\prime}}, l_{j^{\prime}}\right)$, the function $\chi_{2}$ should be taken to be $\chi_{2}=\eta_{i^{\prime}} \otimes \eta_{j^{\prime}}$; in the expression $\widehat{\chi_{2 n} \psi_{2 n}}\left(l_{1}, \ldots, l_{n},-l_{n}, \ldots,-l_{1}\right)$, the function $\chi_{2 n}$ should be taken to be $\chi_{2 n}=\eta_{1} \otimes \ldots \eta_{n} \otimes \eta_{n} \otimes \ldots \eta_{1}$, etc. 
We would now like to argue that the right side of inequality (38) is rapidly decaying in directions for which all $l_{i}$ are in some conic neighborhood of $\left(V_{-}\right)_{x_{i}}$, with at least one $l_{i} \neq 0$, provided the support of the functions $\eta_{i}$ is localized sharply enough around points $x_{i}$. For this, we first look at the terms in the sum on the right side of inequality (38). Each term in this sum contains at least one factor of either the form $\widehat{\chi_{2} \omega_{2}}\left(l_{i}, l_{j}\right)$ or $\widehat{\chi_{2} \omega_{2}}\left(-l_{j^{\prime}},-l_{i^{\prime}}\right)$, where $(i, j) \in P$ or $\left(i^{\prime}, j^{\prime}\right) \in P^{\prime}$. Provided that not all the covectors $l_{i}, l_{j}, l_{i^{\prime}}, l_{j^{\prime}}$ occurring in these factors are zero, these factors give us rapid decay of the corresponding term in the sum. This is because, by the Hadamard property of $\omega_{2}$,

$$
\left|\widehat{\chi_{2} \omega_{2}}\left(k_{1}, k_{2}\right)\right| \leq C_{N}\left(1+\left|k_{1}\right|+\left|k_{2}\right|\right)^{-N}
$$

for all $N$ and all directions $\left(k_{1}, k_{2}\right)$ in some conic neighborhood of $\left(V_{+}\right)_{x_{1}} \times\left(V_{+}\right)_{x_{2}} \cup$ $\left(V_{-}\right)_{x_{1}} \times\left(V_{-}\right)_{x_{2}}$, provided $\chi_{2}$ is localized sufficiently sharply around $\left(x_{1}, x_{2}\right)$. If all the $l_{i}, l_{j}, l_{i^{\prime}}, l_{j^{\prime}}$ occurring in the factors $\widehat{\chi_{2} \omega_{2}}\left(l_{i}, l_{j}\right)$ or $\widehat{\chi_{2} \omega_{2}}\left(-l_{j^{\prime}},-l_{i^{\prime}}\right)$ are zero, then at least one of the covectors $l_{k}$ with $k \notin P$ and at least one of the covectors $l_{k^{\prime}}$ with $k^{\prime} \notin P^{\prime}$ must be nonzero, since otherwise all the $l_{i}$ would be zero. Let us first assume that $P$ is not the empty set. Then we have

$$
\left|\chi_{n-2|P|} \Psi_{n-2|P|}\left(-l_{k_{1}},-l_{k_{2}}, \ldots,-l_{k_{n-2|P|}}\right)\right| \leq C_{N}\left(1+\sum_{k \notin P}\left|l_{k}\right|\right)^{-N}
$$

for all $N$ and suitable constants $C_{N}$, since the distributions $\Psi_{n-2|P|}$ are smooth by the inductive assumption. If $P=\emptyset$, then $P^{\prime}$ is not the empty set, and we get an estimate of

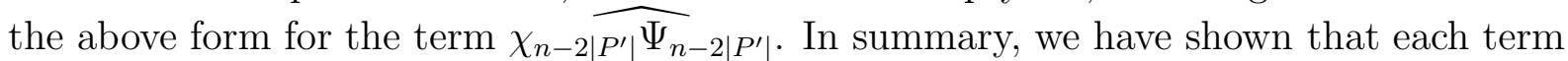
in the sum on the right side of (38) contains at least one factor which is rapidly decaying. Therefore the whole sum is rapidly decaying in directions such that either all $l_{i} \in\left(V_{+}\right)_{x_{i}}$ or all $l_{i} \in\left(V_{-}\right)_{x_{i}}$ and not all $l_{i}=0$, provided the functions $\eta_{i}$ are localized sufficiently sharply around the points $x_{i}$.

By Lemma [II.5, the first term on the right side of (38) is rapidly decaying in directions for which all $l_{i}$ are in a conic neighborhood of $\left(V_{-}\right)_{x_{i}}$, provided the functions $\eta_{i}$ are localized sharply enough around $x_{i}$. Hence, we have altogether found that

$$
\widehat{\mid \chi_{n} \Psi_{n}}\left(l_{1}, l_{2}, \ldots, l_{n}\right) \mid \leq C_{N}\left(1+\sum_{i}\left|l_{i}\right|\right)^{-N}
$$

in directions for which all $l_{i}$ are in a neighborhood of $\left(V_{-}\right)_{x_{i}}$, provided the functions $\eta_{i}$ are localized sharply enough around $x_{i}$. Now, since

$$
\left(: \varphi^{\otimes n}(t): \omega\right)^{*}=: \varphi^{\otimes n}(\bar{t}): \omega
$$

for all testfunctions $t$, the distributions $\Psi_{n}$ are real, in the sense that $\overline{\Psi_{n}(t)}=\Psi_{n}(\bar{t})$ for all testfunctions $t$. Hence, $\left|\widehat{\chi_{n} \Psi_{n}}\left(l_{1}, \ldots, l_{n}\right)\right|=\left|\widehat{\chi_{n} \Psi_{n}}\left(-l_{1}, \ldots,-l_{n}\right)\right|$, and the inequality 
(41) must therefore also hold if all the $l_{i}$ in that inequality are replaced by $-l_{i}$, that is, (41) must also hold in directions for which all $l_{i}$ are in some neighborhood of the cones $\left(V_{+}\right)_{x_{i}}$. Therefore, since we already know that $\mathrm{WF}\left(\Psi_{n}\right) \subset V_{+}^{n} \cup V_{-}^{n}$, we get from this that the distribution $\chi_{n} \Psi_{n}$ has in fact no singular directions at all, provided the supports of $\eta_{i}$ are sufficiently sharply localized around $x_{i}$. But this implies that $\mathrm{WF}\left(\Psi_{n}\right)=\emptyset$, as we wanted to show.

We next prove (ii). Let us thus assume $\psi$ is a state on $\mathcal{A}$ for which $\psi_{2}$ is Hadamard and for which $\psi_{n}^{T}$ are smooth for all $n \neq 2$. By Lemma [II.1, this implies that the distributions $\Psi_{n}$ are smooth. We are thus allowed to define an action of $\psi$ on elements of $\mathcal{W}$ by the formula

$$
\psi\left(: \varphi^{\otimes n}(t): \omega\right) \equiv \Psi_{n}(t)
$$

for all $t \in \mathcal{E}_{n}^{\prime}$ and all $n$. It is easily checked that this formula defines a linear, normalized and continuous functional on $\mathcal{W}$ which extends the action of $\psi$ on $\mathcal{A}$. Moreover, this functional is also positive, since it is continuous and positive on $\mathcal{A}$, which is a dense subspace of $\mathcal{W}$.

\section{Appendix}

\section{IV.1 Proof of formula (22)}

Let $\left\{h_{1}, h_{2}, \ldots, h_{n}, \ldots\right\}$ denote some hierarchy of symmetric distributions and let

$$
H(f)=1+\sum_{n \geq 1} \frac{i^{n}}{n !} h_{n}(f, f, \ldots, f)
$$

be the corresponding generating functional. Then the "linked cluster theorem" (see e.g. [17, pp 125]) states that the corresponding truncated distributions are given by

$$
h_{n}^{T}\left(f_{1}, f_{2}, \ldots, f_{n}\right)=\left.\frac{\partial^{n}}{i^{n} \partial t_{1} \partial t_{2} \ldots \partial t_{n}} \ln H\left(\sum_{i} t_{i} f_{i}\right)\right|_{t_{1}=t_{2}=\cdots=0} .
$$

We would like to apply this result to the hierarchies $\left\{\psi_{1}, \psi_{2}, \ldots, \psi_{n}, \ldots\right\}$ and $\left\{\omega_{1}, \omega_{2}, \ldots, \omega_{n}, \ldots\right\}$ of the $n$-point functions of the states $\psi$ and $\omega$. However, these are not symmetric and therefore the linked cluster theorem is not directly applicable. Instead, we first apply the linked cluster theorem to the hierarchies of symmetrized $n$-point functions, 
$\left\{\psi_{1}^{S}, \psi_{2}^{S}, \ldots, \psi_{n}^{S}, \ldots\right\}$ and $\left\{\omega_{1}^{S}, \omega_{2}^{S}, \ldots, \omega_{n}^{S}, \ldots\right\}$, where the superscript " $S$ " stands for symmetrization. This gives us

$$
\begin{aligned}
{\left[\left(\psi_{n}^{S}\right)^{T}-\left(\omega_{n}^{S}\right)^{T}\right]\left(f_{1}, \ldots, f_{n}\right) } & =\left.\frac{\partial^{n}}{i^{n} \partial t_{1} \ldots \partial t_{n}}\left[\ln \psi\left(e^{i \varphi\left(\sum_{i} t_{i} f_{i}\right)}\right)-\ln \omega\left(e^{i \varphi\left(\sum_{i} t_{i} f_{i}\right)}\right)\right]\right|_{t_{1}=t_{2}=\cdots=0} \\
& =\left.\frac{\partial^{n}}{i^{n} \partial t_{1} \ldots \partial t_{n}} \ln \psi\left(G\left(\sum_{i} t_{i} f_{i}\right)\right)\right|_{t_{1}=t_{2}=\cdots=0}
\end{aligned}
$$

where we have used the definition of $G(f)$, Eq. (14), as well as the relation $\omega\left(e^{i \varphi(f)}\right)=$ $e^{-\frac{1}{2} \omega_{2}(f, f)}$, which holds because $\omega$ is quasi-free. The desired relation (22) then follows if we can show that

$$
\left(\psi_{n}^{S}\right)^{T}-\left(\omega_{n}^{S}\right)^{T}=\psi_{n}^{T}-\omega_{n}^{T}
$$

for all $n$. The demonstration of (47) makes up the rest of this subsection.

Relation (47) can be checked immediately for $n=1$ and $n=2$. For $n \geq 3$ it reduces to

$$
\left(\psi_{n}^{S}\right)^{T}=\psi_{n}^{T}
$$

since $\omega_{n}^{T}=\left(\omega_{n}^{S}\right)^{T}=0$ for $n \geq 3$. In order to see (48), we first note that the truncated $n$-point functions of any state on $\mathcal{A}$ are symmetric for $n \geq 3, \psi_{n}^{T}=\left(\psi_{n}^{T}\right)^{S}$, as one can show by a straightforward inductive argument using the commutation relation (3) for the free field. Eq. (48) thus follows from the fact that for any hierarchy $\left\{h_{1}, h_{2}, \ldots, h_{n}, \ldots\right\}$ (not necessarily symmetric) there holds

$$
\left(h_{n}^{T}\right)^{S}=\left(h_{n}^{S}\right)^{T}
$$

for all $n$. To see this, we argue as follows. Let $P=\left\{r_{1}, \ldots, r_{k}\right\}$, and let $Q\left(r_{j}\right)$ denote the set of all permutations of $r_{j} \equiv\left(r_{j}(1), \ldots, r_{j}\left(n_{j}\right)\right)$ where $n_{j}=\left|r_{j}\right|$. Let

$$
Q(P)=\left\{\sigma_{1} \cdots \sigma_{k} \mid \sigma_{j} \in Q\left(r_{j}\right) \text { for } j=1, \ldots, k\right\} .
$$

Let $\mathcal{I}\left(n_{1}, \ldots, n_{k}\right)$ denote the subset of partitions which has $k$ members $\left\{r_{1}, \ldots, r_{k}\right\}$ such that for each $i=1, \ldots, k,\left|r_{j}\right|=n_{j}$ for all $j$. For any fixed $P \in \mathcal{I}\left(n_{1}, \ldots, n_{k}\right)$, we then get

$$
\begin{aligned}
\left(\prod_{r \in P} h_{|r|}\right)^{S}\left(x_{1}, \ldots, x_{n}\right) & =\frac{1}{n !} \sum_{P^{\prime} \in \mathcal{I}\left(n_{1}, \ldots, n_{k}\right)} \sum_{\sigma \in Q\left(P^{\prime}\right)}\left(\prod_{r_{j} \in P^{\prime}} h_{n_{j}}\right)\left(x_{\sigma(1)}, \ldots, x_{\sigma(n)}\right) \\
& =\frac{n_{1} ! \cdots n_{k} !}{n !} \sum_{P^{\prime} \in \mathcal{I}\left(n_{1}, \ldots, n_{k}\right)} \prod_{r_{j} \in P^{\prime}} h_{n_{j}}^{S}\left(x_{r_{j}(1)}, \ldots, x_{r_{j}\left(n_{j}\right)}\right) .
\end{aligned}
$$


(Note that the left hand side is independent of $P \in \mathcal{I}\left(n_{1}, \ldots, n_{k}\right)$.) From this we conclude that

$$
\begin{aligned}
\left(\sum_{P \in \mathcal{I}_{n}} \prod_{r \in P} h_{|r|}\right)^{S}\left(x_{1}, \ldots, x_{n}\right) & =\sum_{\left\{n_{1}, \ldots, n_{k}\right\}} \sum_{P \in \mathcal{I}\left(n_{1}, \ldots, n_{k}\right)}\left(\prod_{r \in P} h_{|r|}\right)^{S}\left(x_{1}, \ldots, x_{n}\right) \\
& =\sum_{\left\{n_{1}, \ldots, n_{k}\right\}} \sum_{P^{\prime} \in \mathcal{I}\left(n_{1}, \ldots, n_{k}\right)} \prod_{r_{j} \in P^{\prime}} h_{n_{j}}^{S}\left(x_{r_{j}(1)}, \ldots, x_{r_{j}\left(n_{j}\right)}\right) \\
& =\sum_{P \in \mathcal{I}_{n}} \prod_{r \in P} h_{n_{j}}^{S}\left(x_{r_{j}(1)}, \ldots, x_{r_{j}\left(n_{j}\right)}\right)
\end{aligned}
$$

where the sum $\sum_{\left\{n_{1}, \ldots, n_{k}\right\}}$ is over all possible set of positive integers such that $n_{1}+\ldots+n_{k}=$ $n$.

We now use this formula to prove Eq. (49). Suppose that this equation is true for $1,2, \ldots, n$. We now show that it must also be true for $n+1$.

By the induction hypothesis, for any $P \in \mathcal{I}_{n+1}$ which is not $P_{0}=\{(1,2, \ldots, n+1)\}$ and any $r \in P$, we have that

$$
\left(h_{|r|}^{S}\right)^{T}\left(x_{r(1)}, \ldots, x_{r(|r|)}\right)=\left(h_{|r|}^{T}\right)^{S}\left(x_{r(1)}, \ldots, x_{r(|r|)}\right)
$$

Hence,

$$
\begin{aligned}
\left(h_{n+1}^{S}\right)^{T}\left(x_{1}, \ldots, x_{n}\right) & =h_{n+1}^{S}\left(x_{1}, \ldots, x_{n+1}\right)-\sum_{P \in \mathcal{I}_{n+1}, P \neq P_{0}} \prod_{r \in P}\left(h_{|r|}^{S}\right)^{T}\left(x_{r(1)}, \ldots, x_{r(|r|)}\right) \\
& =h_{n+1}^{S}\left(x_{1}, \ldots, x_{n+1}\right)-\sum_{P \in \mathcal{I}_{n+1}, P \neq P_{0}} \prod_{r \in P}\left(h_{|r|}^{T}\right)^{S}\left(x_{r(1)}, \ldots, x_{r(|r|)}\right) \\
& =h_{n+1}^{S}\left(x_{1}, \ldots, x_{n+1}\right)-\left(\sum_{P \in \mathcal{I}_{n+1}, P \neq P_{0}} \prod_{r \in P} h_{|r|}^{T}\right)^{S}\left(x_{1}, \ldots, x_{n+1}\right) \\
& =\left(h_{n+1}^{T}\right)^{S}\left(x_{1}, \ldots, x_{n+1}\right),
\end{aligned}
$$

where in the first line we have used the definition of the truncated $n$-point functions, in the second line we have used the induction hypothesis, and where in the third line we have applied formula (50), applied to the hierarchy $\left\{h_{1}^{T}, h_{2}^{T}, \ldots, h_{n}^{T}, \ldots\right\}$. This completes the induction.

\section{IV.2 Proof of Lemma III.2}

Let $\Gamma$ be a closed conic subset of $T^{*} M^{n} \backslash\left(V_{+}^{n} \cup V_{-}^{+}\right)$and let $\left\{t_{\kappa}\right\}$ be a sequence of smooth functions on $M^{n}$, whose support is contained in some compact subset of $M^{n}$ for all $\kappa$, and which converges to some $t$ in the sense of $\mathcal{D}_{\Gamma}^{\prime}$. Then, the sequences $A_{\kappa}=: \varphi^{\otimes n}\left(t_{\kappa}\right):_{\omega}$ 
by definition converges in $\mathcal{W}$. Therefore, since the state $\psi$ is assumed to be continuous on $\mathcal{W}, \psi\left(A_{\kappa}\right)=\Psi_{n}\left(t_{\kappa}\right)$ is a convergent sequence for $\kappa \rightarrow \infty$. We need to show that this implies that $\operatorname{WF}\left(\Psi_{n}\right) \subset V_{+}^{n} \cup V_{-}^{n}$. This immediately follows from the following general result.

Lemma IV.1. Let $u \in \mathcal{D}^{\prime}\left(\mathbb{R}^{n}\right)$ and let $\Gamma$ be a closed conic set in $\mathbb{R}^{n} \times\left(\mathbb{R}^{n} \backslash\{0\}\right)$. Assume that $u$ has the following property. For every sequence of smooth functions $\left\{f_{\kappa}\right\}$ such that $f_{\kappa} \rightarrow f$ in $\mathcal{D}_{\Gamma}^{\prime}\left(\mathbb{R}^{n}\right)$ and such that $\operatorname{supp}\left(f_{\kappa}\right) \subset K$, with $K$ a compact subset of $\mathbb{R}^{n}$, we have that $\left\{u\left(f_{\kappa}\right)\right\}$ is a convergent sequence. Then $\{0\} \notin \mathrm{WF}(u)+\Gamma$.

Proof. Let $A$ be a properly supported pseudo differential operator and with $\mu \operatorname{supp}(A) \subset$ $\Gamma$. Then, if $\left\{f_{\kappa}\right\}$ is any sequence of distributions on $\mathbb{R}^{n}$ converging weakly to some $f \in \mathcal{D}^{\prime}\left(\mathbb{R}^{n}\right)$ in the sense of distributions, it follows that $A f_{\kappa} \rightarrow A f$ in the sense of $\mathcal{D}_{\Gamma}^{\prime}\left(\mathbb{R}^{n}\right)$ and that $\operatorname{supp}\left(A f_{\kappa}\right) \subset K^{\prime}$, where $K^{\prime}$ is some compact subset of $\mathbb{R}^{n}$. Therefore, the sequence $u\left(A f_{\kappa}\right)=A^{t} u\left(f_{\kappa}\right)$ is convergent, by the assumption of the lemma, where $A^{t}$ is the formal adjoint of the pseudo differential operator $A$. We claim that it follows from this that $A^{t} u$ is in fact smooth. Assuming for the moment that this has been shown to be true, we get from the characterization [7] of the wave front set that

$$
\mathrm{WF}(u)=\bigcap_{B u \in C^{\infty}} \operatorname{char}(B) \subset \bigcap_{\mu \operatorname{supp}(A) \subset \Gamma} \operatorname{char}\left(A^{t}\right)=\bigcap_{\mu \operatorname{supp}(A) \subset \Gamma}-\operatorname{char}(A)=T^{*} \mathbb{R}^{n} \backslash(-\Gamma),
$$

because the set of properly supported pseudo differential operators $A$ with $\mu \operatorname{supp}(A) \subset \Gamma$ contains elements whose characteristic, $\operatorname{char}(A)$, is contained in an arbitrarily small conic neighborhood of $T^{*} \mathbb{R}^{n} \backslash \Gamma$. Since the set $T^{*} \mathbb{R}^{n} \backslash(-\Gamma)$ contains no element $(x, k)$ such that $k+k^{\prime}=0$ for some $\left(x, k^{\prime}\right) \in \Gamma$, this then proves the lemma.

It thus remains to be shown that the compactly supported distribution $v=A^{t} u$ is smooth. This would immediately follow if the Fourier transform, $\widehat{v}(k)$ was rapidly decaying. Let us assume on the contrary that $\widehat{v}(k)$ is not rapidly decaying. We will show that this assumption is in contradiction with the fact that the sequence $v\left(f_{\kappa}\right)$ is converging for any weakly convergent sequence of distributions $f_{\kappa}$. So let us assume that $\widehat{v}(k)$ is not rapidly decaying. Then there exists an $N$, a positive constant $C$, and a sequence of $k_{j} \in \mathbb{R}^{n}$ with $\left|k_{j}\right|>j$ such that

$$
\left|\widehat{v}\left(k_{j}\right)\right| \geq C\left(1+\left|k_{j}\right|\right)^{-N} \text { for all } j .
$$

(We adopt from now on the convention that all strictly positive constants appearing in the various inequalities are denoted by the same letter, $C$, irrespective of their numerical value.) Let now $e \in \mathbb{R}^{n}$ with $|e|=1$ and let $t$ be a real number with $|t| \leq r_{j}$, where 
$r_{j}>0$. Then we have

$$
\begin{aligned}
\left|\widehat{v}\left(k_{j}+t e\right)-\widehat{v}\left(k_{j}\right)\right| & \leq \int_{0}^{r_{j}}\left|e \cdot \partial \widehat{v}\left(k_{j}+t e\right)\right| d t \\
& \leq \int_{0}^{r_{j}} C\left(1+\left|k_{j}\right|+|t|\right)^{M} d t \\
& \leq C r_{j}\left(1+\left|k_{j}\right|+\left|r_{j}\right|\right)^{M}
\end{aligned}
$$

for some $M$. This is easily seen to imply

$$
|\widehat{v}(l)| \geq C\left(1+\left|k_{j}\right|\right)^{-N} \text { for all } j
$$

and all $l \in B_{r_{j}}\left(k_{j}\right)$, where $r_{j}=C\left(1+\left|k_{j}\right|\right)^{-N-M}$ and $C>0$ is some sufficiently small constant. Let now $\rho_{j}$ be a function of compact support which is equal to one on $B_{r_{j}}\left(k_{j}\right)$ and which is zero outside $B_{2 r_{j}}\left(k_{j}\right)$ and which satisfies $0 \leq \rho_{j}(l) \leq 1$ for all $l \in \mathbb{R}^{n}$. Then, for any natural number $\kappa$ we define a smooth function $f_{\kappa}$ on $\mathbb{R}^{n}$ by

$$
\widehat{f}_{\kappa}(l)=\overline{\widehat{v}(-l)} \sum_{j=1}^{\kappa}\left(1+\left|k_{j}\right|\right)^{L} \rho_{j}(-l),
$$

where $L$ is a constant to be chosen below. Note that, since

$$
\left|\left(1+\left|k_{j}\right|\right)^{L} \rho_{j}(l)\right| \leq C(1+|l|)^{L} \quad \text { for all } j,
$$

the functions $f_{\kappa}$ converge weakly to some distribution $f$. By what we have already said above, this means that the sequence $v\left(f_{\kappa}\right)$ should converge as $\kappa \rightarrow \infty$. We will now show that this sequence in fact manifestly diverges, leading thus to the desired contradiction. Inserting the definition of $f_{\kappa}$, we get

$$
\begin{aligned}
v\left(f_{\kappa}\right) & =\int_{\mathbb{R}^{n}} \widehat{v}(l) \widehat{f}_{\kappa}(-l) d^{n} l \\
& =\sum_{j=1}^{\kappa} \int_{\mathbb{R}^{n}}|\widehat{v}(l)|^{2}\left(1+\left|k_{j}\right|\right)^{L} \rho_{j}(l) d^{n} l \\
& \geq C \sum_{j=1}^{\kappa} \int_{B_{r_{j}}\left(k_{j}\right)}\left(1+\left|k_{j}\right|\right)^{-2 N+L} d^{n} l \\
& \geq C \sum_{j=1}^{\kappa}\left(1+\left|k_{j}\right|\right)^{-2 N+L} r_{j}^{n} \\
& \geq C \sum_{j=1}^{\kappa}\left(1+\left|k_{j}\right|\right)^{-2 N-n(N+M)+L} .
\end{aligned}
$$

If $L$ is now chosen large enough, then the right side of the above inequality diverges for $\kappa \rightarrow \infty$, since $k_{j}>j$. 


\section{References}

[1] See for example the lectures given at the 1975 Erice summer school, collected in: "Renormalization Theory," G. Velo and A. S. Wightman, eds., NATO ASI Series C 23, Reidel, Dodrecht, 1976

[2] T. S. Bunch: "BPHZ renormalization of $\lambda \Phi^{4}$ field theory in curved space-times," Ann. of Phys. 131, 118 (1981)

[3] T. S. Bunch, P. Panangaden and L. Parker: "On renormalization of $\lambda \Phi^{4}$ in curved space-time I," J. Phys. A: Math. Gen. 13, 901-918 (1980), "On renormalization of $\lambda \Phi^{4}$ in curved space-time II," J. Phys. A: Math. Gen. 13, 919-932 (1980)

[4] R. Brunetti, K. Fredenhagen and M. Köhler: "The microlocal spectrum condition and Wick polynomials on curved spacetimes," Commun. Math. Phys. 180, 633-652 (1996)

[5] R. Brunetti and K. Fredenhagen: "Microlocal Analysis and Interacting Quantum Field Theories: Renormalization on physical backgrounds," Commun. Math. Phys. 208, 623-661 (2000)

[6] S. Hollands and R. M. Wald: "Local Wick polynomials and time ordered products of quantum fields in curved spacetime," Commun. Math. Phys., in print, [grqc/0103074.

[7] L. Hörmander: "The Analysis of Linear Partial Differential Operators I-IV", Springer-Verlag, Berlin 1985

[8] S. Hollands and R. M. Wald: work in progress.

[9] R. M. Wald: "Quantum Field Theory in Curved Spacetime and Black Hole Thermodynamics," The University of Chicago Press, Chicago 1994

[10] B. S. Kay and R. M. Wald: "Theorems on the uniqueness and thermal properties of stationary, nonsingular, quasifree states on spacetimes with a bifurcate Killing horizon," Phys. Rep. 207, 49 (1991)

[11] M. J. Radzikowski: "Micro-Local Approach to the Hadamard condition in QFT on Curved Space-Time," Commun. Math. Phys. 179, 529-553 (1996)

[12] M. Dütsch and K. Fredenhagen: "Algebraic quantum field theory, perturbation theory, and the loop expansion," hep-th/0001129]; "Perturbative algebraic field theory, and deformation quantization," [hep-th/0101079]. 
[13] C. Lüders and J. Roberts: "Local quasiequivalence and adiabatic vacuum states," Commun. Math. Phys. 134, 29 (1990).

[14] S. Hollands: "The Hadamard condition for Dirac fields and adiabatic states on Robertson-Walker spacetimes," Commun. Math. Phys. 216, 635 (2001) [grqc/9906076.

[15] W. Junker: in preparation.

[16] R. Verch: "Local definiteness, primarity and quasiequivalence of quasifree Hadamard quantum states in curved space-time," Commun. Math. Phys. 160, 507 (1994).

[17] Handbuch der Physik, Band XII, "Thermodynamik der Gase," Springer-Verlag 1958 